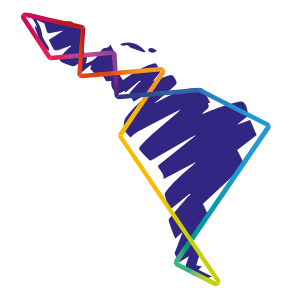

\title{
Los sistemas de cargo en una comunidad migrante translocal de la mixteca poblana
}

\author{
Cargo systems in a translocal \\ migrant community in la mixteca \\ poblana
}

\section{Os sistemas de carga em uma comunidade de migrante translocal na mixteca poblana}

\author{
Gustavo López Ángel ${ }^{1}$ \\ Alex Munguía Salazar ${ }^{2}$ \\ Pedro Manuel Rodríguez Suárez ${ }^{3}$
}

\begin{abstract}
Resumen
El sistema de gobernanza de las comunidades indígenas difiere de las nociones tradicionales, porque, a diferencia de su condición pasiva, esta descansa en un constante ejercicio de transformación con dinámicas derivadas de la migración interna e internacional. La ciudadanía sustantiva tradicional ha reposado sobre una concepción de comunidad homogénea, con sistemas de pertenencia únicos, sin reparar o incluir, en el análisis, la diversidad de actores y procesos que existen en su interior. Por ello, el tránsito hacia la ciudadanía multicultural es una de las próximas tareas en la construcción de políticas públicas del Estado mexicano.
\end{abstract}

Palabras claves: gobernanza, comunidades indígenas, translocal.

1 Profesor tiempo completo, asociado al Instituto de Investigaciones Jurídicas y Políticas de la Benemérita Universidad Autónoma de Puebla (BUAP), México. ORCID: https://orcid.org/0000-0001-8827-2200

2 Dr. en Ciencias Políticas y Sociales por la Universidad Nacional Autónoma de México. Profesor investigador de la BUAP, México. ORCID: https://orcid.org/0000-0001-9030-2149

3 Profesor tiempo completo, titular B en la Licenciatura de Relaciones Internacionales de la BUAP. Integrante del Sistema Nacional de Investigadores, Conacyt, México, nivel 2. ORCID: https://orcid.org/0000-0002-4398-1625 


\begin{abstract}
The governance system of indigenous communities differs from traditional notions, because unlike its passive condition, it rests on a constant exercise of transformation with dynamics derived from internal and international migration. The traditional substantive citizenship has rested on a homogeneous conception of community, with unique membership systems, without repairing or including, in the analysis, the diversity of actors and processes that exist within it. For this reason, the transition towards multicultural citizenship is one of the tasks ensuing the construction of public policies of the Mexican State.
\end{abstract}

Keywords: Governance; Indigenous communities; Translocal.

\title{
Resumo
}

O sistema de governança das comunidades indígenas difere das noções tradicionais, porque, diferentemente de sua condição passiva, repousa em um constante exercício de transformação com dinâmica derivada da migração interna e internacional. A cidadania substantiva tradicional baseou-se em uma concepção homogênea de comunidade, com sistemas de associação únicos, sem reparar ou incluir, a análise, a diversidade de atores e processos que nela existem. Por esse motivo, a transição para a cidadania multicultural é uma das próximas tarefas na construção de políticas públicas do Estado mexicano.

Palavras-chave: Governança; Comunidades indígenas; Translocal.

\section{Introducción}

En este ensayo, a partir del testimonio de los actores sociales, se expondrán los cambios, continuidades e innovaciones de la gobernanza, a través del sistema de cargos en una comunidad indígena mixteca migrante del sur del estado de Puebla, México. Se analiza, desde una perspectiva diacrónica, el papel que juegan los procesos organizativos translocales generados por la migración en los mecanismos de nombramiento, en la asamblea comunitaria local. Tal nombramiento se ve definido por nuevos perfiles y atributos requeridos para quienes ocupan el cargo. En ese sentido, nuestro referente etnográfico nos permitirá dilucidar el papel de la Asociación Micaltepecana en la continuidad, la innovación y el cambio de estas nuevas lógicas que aglutinan a los integrantes, independientemente de su lugar de residencia, rompiendo con las prácticas esencialistas que precisaban la pertenencia comunitaria.

Nuestro referente etnográfico es El Rosario Micaltepec. Administrativamente, es una junta auxiliar ${ }^{4}$ perteneciente al municipio de Petlalcingo, en el sur del estado

4 Las juntas auxiliares son comunidades que fueron pueblos vecinos, rancherías o ejidos e incluso municipios los cuales fueron absorbidos por una entidad municipal mayor y que ahora forman parte de la mancha urbana municipal.

$122 \begin{aligned} & \text { Revista Latinoamericana de Derechos Humanos } \\ & \text { Volumen 31 (1), I Semestre 2020 } \\ & \text { ISSN: 1659-4304 • EISSN: 2215-4221 }\end{aligned}$ 
de Puebla, México. La figura jurídica administrativa de junta auxiliar acota los ámbitos y funciones que puede desempeñar, en aras de establecer un control desde la presidencia del municipio hacia los pueblos indígenas, por parte de los mestizos. Pese a ello, en el terreno de los hechos, se mantiene la autonomía en algunas esferas, como la mecánica para designar a las autoridades, sus funciones y su permanencia, lo cual corresponde a los sistemas normativos indígenas ${ }^{5}$. La asamblea del pueblo constituye la máxima autoridad; en ella se deciden los aspectos más relevantes de la vida de aquel. Esta forma de administración la encontramos en otros pueblos mixtecos como San Jerónimo del Progreso, Oaxaca (Besserer y Kearney, 2004).

Existe una interdependencia entre las autoridades políticas y las religiosas; ambas comparten elementos cuya función es el servicio para el mantenimiento del orden. Son aproximadamente 100 cargos, los cuales son distribuidos en 70 jefes de familia (o 70 "familias activas"), por lo que es necesario que varios integrantes del grupo doméstico (siempre y cuando sean mayores de edad) ejerzan algún cargo o cumplan con el tequio. ${ }^{6}$ En la memoria de los rosareños:

[...] siempre ha sido así, cambian las personas y las cosas qué hacer, pero los cargos igual; los comités les decimos, la forma pues: [... $]^{7}$ porque lo mismo me dice (le decía) mi papá, no, que me dieron un cargo, que, pues me toca la acción católica, o me toca el comité de la carrera de caballos o me toca hacer esto por la pelea de gallos o qué sé yo ( H. Flores, entrevista 2018).

La memoria de los sujetos no recapitula acerca de los procesos de cambio en el repertorio del sistema de cargos; la irrupción de las instituciones incrementó el número de estos. Un ejemplo de ello son los comités de agua potable, salud, educación, etc. La comunidad se ha visto sometida a un doble juego: el desplome demográfico ha incrementado las obligaciones en el sistema de cargos y las instituciones gubernamentales han contribuido también a su aumento.

\section{Estructura organizativa y mecánica para la designación de cargos}

En cuanto a la estructura, el sistema no ha cambiado, las variaciones han sido dependiendo de las nuevas acciones que en el pueblo haya que realizar, debido al

5 "Los sistemas normativos de las comunidades indígenas en Chiapas", documento, INI-Chiapas, San Cristóbal de las Casas, 1998.

6 La palabra proviene del náhuatl, tequitl como tributo y tequitiliztli como trabajo. Se le define, entonces, como obra o tributo. En México, se conoce como tequio a la faena o trabajo colectivo no remunerado que todo vecino de un pueblo debe a su comunidad. Es un uso y una costumbre de origen indígena, que con diversos matices y fuerza continúa su arraigo en varias zonas de este país. Esther Hernández (1996). Vocabulario en lengua castellana y mexicana de fray Alonso de Molina. España: Consejo Superior de Investigaciones Científicas. Pp. $162-163$.

7 El uso de iniciales busca mantener el anonimato de las personas entrevistadas. 
crecimiento de infraestructura; por ejemplo, la construcción de la red del drenaje (se agrega un nuevo comité). Cada comité tiene un presidente, un tesorero, vocales y serviciales. El número de integrantes depende del tipo de comité o del trabajo que se vaya a ejecutar. Hay diez comités: Acción Católica, Agua Potable, Albergue Escolar, Drenaje, Comité del DIF, Escuela Primaria, Luz Eléctrica, Pequeña Propiedad, Preescolar y Salud.

Existe una jerarquización en los cargos, el más alto lo ejerce el presidente auxiliar, seguido por el presidente del comité de la Pequeña Propiedad (o Unión de Pequeños Propietarios) y después por el presidente de Acción Católica. Los restantes cargos tienen una importancia similar. La manera de referirse a una autoridad es como "comité": "Don Pedro J. es comité ahorita" o "está de comité"; esta metonimia alude a la responsabilidad y, a la vez, al poder que implica el ejercicio del cargo.

La presidencia auxiliar está conformada por un presidente, un tesorero y cinco regidores (Gobernación, Educación, Hacienda, Obras Públicas y Salud) todos ellos con sus suplentes; también, por un comandante de policía, un subcomandante, ocho policías, un juez de paz y seis serviciales (el número varía).

Las autoridades son elegidas en la asamblea, máxima autoridad del pueblo. Se convoca cuando hay algún asunto de interés general, principalmente, cuando hay elección de autoridades. El encargado de convocar es el presidente auxiliar; este comunica a los serviciales, quienes llamarán a los integrantes del cabildo y, después, por el altavoz se llama al pueblo. La asamblea es realizada principalmente por las noches, cuando ya se ha regresado del campo. El anuncio se repite varias veces mediante las bocinas colocadas en la presidencia auxiliar municipal, dando a conocer el orden del día:

La invitación se hace mediante un perifoneo, ya se convoca con anticipación, una semana, cuatro o cinco días antes se da un aviso otro al siguiente día, y otro, y ya llegada la fecha se reúne la comunidad, ya saben.

Empezamos cuando más de la mitad ya está, pero normalmente llegan puntuales. Lo que pasa es que desde que empezamos (su periodo) se aclaró todo este asunto de que debemos respetar el tiempo de los demás y se ha dado tolerancia de 15 a 20 minutos, ya media hora -exagerado-, pero ya no como antes que se daban 2, 3 horas y no se juntaba la gente ( T. Cruz. entrevista, 2018).

La asamblea da inicio, generalmente, cuando ya ha llegado la mayor parte de jefes de familia o sus representantes (esposa o hijos mayores). El presidente auxiliar toma la palabra y expresa el motivo de la reunión, el cual ha sido discutido previamente por el cabildo. Después toman la palabra los integrantes del comité o los directamente 
afectados con el problema. Iniciada la discusión, puede prolongarse por varias horas, hasta que se llega a un acuerdo. Las decisiones de la asamblea son respetadas. Si algún acuerdo es incumplido, se hace un llamado de atención y se pone una multa a quienes lo desacatan. La opción es aceptarlo y disculparse ante la autoridad, algunos son detenidos en caso de "que no entiendan" y finalmente pagan la multa o hacen el trabajo que tenían encomendado.

La participación en las asambleas es predominantemente masculina, las mujeres "nomás ahí están, para cumplir, por si su esposo no está"; ellas tienen un papel activo, pero no generalizado, "una que otra toma la palabra, como esta Berna que es bien entrona". "En una reunión, ora sí que todos pueden opinar, pueden dar su punto de vista todos" (T. Cruz. entrevista, 2017).

Para la elección de autoridades, se discute sobre los candidatos posibles y se realiza la votación. No hay ocasión en la cual haya sido elegida una autoridad que no radique en el pueblo, aunque sí ha habido nombramientos en su ausencia (quienes no asisten a la asamblea), como es el caso de la anterior presidenta auxiliar, designada como servicial. El desconocimiento de ser seleccionado autoridad es relativo; de previo, se ha consultado la lista de vecinos y cargos, se ve quién ha estado "desocupado" o ha tenido menos cargos, en el pueblo se comenta quiénes pueden ser candidatos a los tres principales cargos. Una vez nombrado el presidente, este se encarga de elegir a su equipo de trabajo:

Elegimos por consenso, en una reunión, por usos y costumbres, se dan propuestas, se someten a votación. Se hace una terna, depende de la asamblea, a veces es terna, a veces es opción múltiple, depende del acuerdo de la reunión. Las opciones nacen de la inquietud de los mismos vecinos, o sea ellos mismos ora sí que proponen a personas que ellos consideran que pueden servir o que ya necesitan estar ahí o bueno, muchas ocasiones, por decir, alguien nos cae mal y ora sí queremos que quede ahí, o sea, influyen muchas cosas (T. Cruz entrevista, 2017).

Los cargos en el cabildo son de tres años. Algunos vecinos del pueblo manifiestan su inconformidad por lo prolongado del periodo. Formar parte de la presidencia auxiliar altera sus proyectos de vida y, sobre todo, sus ingresos. Debido al desplome demográfico provocado por la migración, la asamblea comunitaria va haciendo adecuaciones. Otro de los inconvenientes es la falta de remuneración, no hay un salario para los integrantes de la presidencia. En el caso del puesto de presidente, se diseñó un mecanismo de relevo, el cual consistía en dividir el ejercicio de la presidencia de forma anual, y lo ocupaban los primeros tres cargos del cabildo; sin embargo, esto no era funcional e intentaron por meses, integrando en el proceso de rotación a todos los regidores y al tesorero. Actualmente, se cubre por días, son tres 
los principales responsables de fungir como autoridades, se dividen los días de la semana, el titular es quien lleva la principal responsabilidad. El presidente auxiliar don Tomás Cruz. nos explica:

La semana nos la dividimos, cada día le toca a uno de los miembros del cabildo, por decirlo, a mí me toca el lunes, a don C. le toca martes y así, aunque yo sea el de la firma, pero nos dividimos cada uno, lo difícil es el tiempo, es el que nos roba bastante, a veces tengo que faltar al trabajo para atender los asuntos de la comunidad. En horario laboral tengo que salirme para poder atender algún asunto, en ocasiones cuando hay problemas fuertes tengo que quedarme a atender el problema y no asistir al trabajo.

Soy profesor, en eso es mi trabajo, porque mi perfil es ingeniero en comunicaciones y electrónica, egresado del Poli. Doy clases en Oaxaca, aquí en San Miguel Ixtapan que ya pertenece a Oaxaca, en la escuela Telesecundaria.

Nosotros laboramos de ocho a dos de la tarde y entonces ya las oficinas aquí en la comunidad las abrimos en la tarde, por acuerdo de asamblea. Nosotros lo expusimos ante la reunión esta situación y la gente está de acuerdo y por el momento no hay ninguna inconformidad, todos los asuntos se han atendido, entonces de ocho a dos voy a laborar y ya en la tarde a partir de las siete de la tarde ya abrimos la presidencia para atender. Si hay necesidad de atender algún asunto en horario laboral, tenemos que dejar el trabajo.

Laboralmente nosotros hemos (él ha) cumplido al cien por ciento, entonces ellos saben también que yo tengo cargo y cuando hay necesidad de faltar, pues es rara vez, pero tengo que avisar: ¿sabe qué?, tengo un asunto, necesito salir, entonces ellos me han brindado esa posibilidad de que pueda yo abandonar, ora sí que por tiempos determinados el trabajo. Tanto el comité de la escuela, como la autoridad municipal me han permitido estar así. Por ese lado por la comunidad y por el otro lado de lo laboral, el supervisor y la delegación sindical de igual manera tienen conocimiento, y le vuelvo a repetir, también ellos nos tienen en un buen concepto y por esa razón, al contrario, nos han tratado de apoyar, a veces me mandan a otro compañero que me apoya con mi grupo y yo ya como que me desahogo un poco más, pero ellos tienen conocimiento de todo (T. Cruz entrevista, 2017).

Con la rotación del cargo, persiste una inconformidad, al no poder ver una obra terminada porque "el otro ya entró en funciones"; en el terreno de la formalidad, el propietario es uno, en los hechos y para el pueblo, el presidente en turno es quien hace las obras, aunque el anterior las haya iniciado: 
[...] gracias a Dios pues está ahí la obra pues que tenemos ahorita, también estábamos con los demás pero pues se iban turnando, de hecho por meses, de hecho yo estuve trabajando desde $[. .$.$] no me acuerdo, la cosa es que eran tres meses y tres meses \mathrm{S}$. (su suplente) y tres meses L. y tres meses C. y así íbamos trabajando y ya en lo del drenaje fue en el periodo de Celedonio., o sea ya estábamos, pero de hecho ellos tenían que traer los papeles para yo firmar como propietaria. Los arquitectos que estaban me venían a buscar a mí y ya luego íbamos a buscar a los otros, o sea ya no estaba yo, pero ya yo lo había iniciado (H. Flores, entrevista, 2018).

Hay un equilibrio en cuanto a los nombramientos, las facciones - cuando menos hay dos- siempre tratan de colocar a un integrante de su grupo en algún cargo, principalmente, en la presidencia auxiliar y en la Pequeña Propiedad. La división por días ha permitido un seguimiento a los trabajos, es decir, una forma de fiscalización, aunque hay disidencias, porque "finalmente no se descansa nada".

\section{Perfiles de los cargos}

Para ocupar algún cargo se requerían ciertas características, por ejemplo, con el fin de ser nombrado presidente auxiliar, se necesita estar casado; ser mayor de edad, originario del pueblo (así se había acostumbrado hasta la designación del actual presidente, el cual no es originario del pueblo), honrado; además, estar bien enterado de los asuntos del pueblo y de la forma como se acostumbra resolverlos; que no haya tenido muchos cargos en el último año; "que tenga palabra" (que sepa expresarse frente a propios y extraños); que "no tenga miedo" de presentarse ante autoridades y personas externas; que lleve "la voz del pueblo"; saber leer y escribir; "que sea bueno con su familia", cualidad mencionada en todo momento, con el argumento de "¿Cómo puede una persona llevar un cargo del pueblo si no es bueno con su familia?" (S. Méndez. entrevista, 2009). Este es el ideal para la persona que detente alguna ocupación; en el terreno de los hechos y con la migración, los criterios se han flexibilizado:

Cualquiera puede ser electo, porque siguen un cierto rol, las personas que ya pasaron las van metiendo a otro cargo, y las que no han estado son las que van metiendo. La mujer soltera no, porque no se le puede dar cargo todavía, si fuera madre soltera, entonces sí, o si fuera algún padre que bueno ya tiene familia, entonces ya entra dentro como vecino activo, que se le llama aquí, pero los jóvenes que todavía no tienen familia, que todavía no forman, no, ni las mujeres.

Se nombra lo que es el ayuntamiento, y se les da un tiempo de uno, dos tres días, a veces de una semana para que ahí a consideración del ayuntamiento nombre a sus 
diversos comités, a consideración de ellos quienes pueden apoyar como presidentes de comités de los otros comités que funcionan aquí (Entrevista a T. Cruz, 2017).

El actual presidente no es originario del pueblo, está avecindado por vínculos matrimoniales, ha vivido alli desde hace ocho años. Pese a la dificultad para los nombramientos, aún los migrantes retornados deben pasar periodos de reinserción a la comunidad, vía acciones y tiempo de permanencia en ella.

Ah, pues como ellos, por ejemplo, la cosa es que ellos están acá (se refiere al matrimonio del actual presidente), pero ya viviendo de años, ahí es como considerándolo que ya es un ciudadano del pueblo porque es de años, pero si tiene días, pues tampoco, o sea, paisanos radicados son como esos que vienen un mes, dos meses y también ya quieren; jéchatela ya! ¿Pues tú quién eres? Si apenas vienes de arrimado, ¿qué hiciste? Y empieza. Y entonces pues no, sí lo tienen que estar viviendo de años aquí (T. Cruz, entrevista, 2017).

De manera formal, los vecinos exentos de cargos son los menores de edad, mujeres solteras, hombres solteros y las viudas de edad avanzada. En la práctica, suelen suplir al jefe de familia en el tequio o en alguna labor de su cargo. También están exentos de los cargos religiosos quienes profesan "otra religión" como los "Testigos de Jehová", sus creencias son "respetadas", pero la situación no es tan clara; se ha discutido en diversas asambleas lo que consideran su falta de compromiso con el pueblo por no aceptar los puestos religiosos:

[...] de hecho, de otra religión, aquí en el pueblo nosotros, ya se ha hecho no la primera junta, sino varias reuniones que es general, que es parte del pueblo, y se les ha dicho pues, a ellos como otra religión, que sí, que ellos están en su derecho de, cualquier persona, de que tengan su religión, pero siempre y cuando cooperar con el pueblo, pero en este caso ellos no cooperan por lo de la fiesta, por la feria, pero de lo que el pueblo les pide que cooperen con la presidencia o sea lo que es parte del pueblo, ellos sí van, dan por ejemplo su tequio, por ejemplo, una cooperación que es parte del pueblo, como por ejemplo lo que es el agua dulce, en el agua potable (T. Cruz, entrevista, 2017).

\section{Los cargos religiosos}

El Comité de Acción Católica tiene como objetivo realizar los trabajos "propios de la iglesia”, como la organización de las fiestas patronales, el orden o asesoramiento de la cofradía y la supervisión de las obras de restauración que se ejecutan en el templo. También, es el encargado de mantener el vínculo religioso con otras comunidades, por medio de las hermandades. 
Los que forman Acción Católica realizan los trabajos que se tienen que desempeñar en la iglesia; cambiar floreros, limpiar, barrer, cambiar las ceras y otras cosas. También se encargan de los movimientos que se requieren para hacer obra en la iglesia como reconstrucción, pintura, adornos del templo. Para cuando llega la fiesta, ellos llevan mucha responsabilidad porque se encargan de los trabajos de la Iglesia y ellos son los que primero tienen que ver con nuestras hermandades.

Ellos proponen a los vecinos quienes van a ser parte de la cofradía de la fiesta. Acción Católica tiene un presidente, su secretario, su tesorero y dos vocales, el cambio de autoridad se hace en Año Nuevo, también se da el cargo en asamblea. El cargo dura dos años, pero a veces puede ser de más, si el presidente de Acción Católica quiere, depende de la voluntad (M. Morales, entrevista, 2018).

La cofradía es un comité que cobra interés de manera especial en los paisanos. El señor A. F. explica cómo se organizaba:

La cofradía es para servir a Dios. Antes se nombraba la cofradía y las cosas eran costeadas por las personas que eran elegidas. Ahora ya tiene ocho o diez años que se cambió por el nombre de comité, antes se nombraba nomás cofradía y las cosas eran costeadas por las personas que la sacaban, eran de siete a diez personas quienes se cooperaban para la fiesta, y para los gastos de la comida y los demás vecinos se nombraban en comisiones para organizar las diversiones y juegos, así nomás, pero salía uno mal porque es mucho gasto [...] (A. Flores. entrevista, 2018)

Con el tiempo se elevaron los precios, entonces los vecinos ya no querían participar, entonces se citó a asamblea donde se tomó acuerdo entre los vecinos y se decidió que ahora en adelante sería de cooperación pareja; casado, viudo o viuda, todos parejo, por familia. Ahora es más fácil porque se hace con la voluntad del pueblo. También dan 40 tortillas diarias durante los tres días que dura la celebración. Cualquiera de los encargados de la cofradía pasa casa por casa a solicitar la cooperación (A. Flores, entrevista, 2018).

La cofradía ha cambiado. Los migrantes, en varias ocasiones, "han pedido la fiesta"; algunos desean, junto con su familia, hacerse cargo del evento, lo cual implica cubrir el total del gasto de bebidas y alimentos consumidos los días que dure la celebración, "para que el pueblo descanse". El actual presidente de Acción Católica, de quien depende la cofradía, convenció a los vecinos - pese a tener el financiamiento de la totalidad de los gastos de la cofradía- de aportar su cooperación (1000 pesos) por familia, "porque aún hay muchos gastos por hacer". Se decidió que el ingreso por cooperaciones no destinadas a la festividad se invirtiera en arreglos para la iglesia: 
[...] algunos paisanos es su gusto pedir la fiesta, aunque no estén, pero están de acuerdo con su familia de aquí y con el pueblo, vienen para la preparación de todo y ponen el dinero de todo, pero siempre terminamos poniendo. Normalmente está formada por dieciséis personas (ocho matrimonios), [...] a ellos les ayudan sus familiares a moler el chile o en la cocina para la comida que se prepara y ofrece durante la fiesta que es para los peregrinos. (P. Díaz, entrevista, 2018)

Los cargos que conforman la cofradía son: primer mayordomo, primer secretario, primer tesorero, primer vocal, con sus respectivos segundos (segundo mayordomo, segundo secretario, etcétera). Todos van acompañados por sus esposas o hijas, en caso de ser viudos. El perfil requerido para ocupar la función es ser casado, originario o avecindado en la comunidad, al margen de la residencia (solo dos casos), así como haber demostrado responsabilidad y seriedad en otros cargos.

En el 2007, se dio un caso, en el que se nombró a una persona radicada en el estado de California. En esta ocasión, no se eligió el comité tradicional de los ocho matrimonios que acompañan al mayordomo. La innovación planteada para modificar la celebración no prosperó, principalmente, por haber modificado el banquete ceremonial (espagueti, tortillas de máquina y no hubo atole de granillo en la madrugada). Esto produjo un descontento generalizado y se acordó no volver a otorgar el cargo a una sola persona, aunque el gasto sí puede cubrirlo una persona o familia, pero acompañada de los matrimonios acostumbrados.

La elección del comité de la cofradía y la toma de acuerdos para la fiesta se hacen de la siguiente manera:

Luego que ya se vio la lista de vecinos, se cita a una asamblea a los vecinos para que se den cuenta a quiénes les va a tocar, se ponen de acuerdo si se acepta el cargo y ahí también se toman los primeros acuerdos para la fiesta. Por ejemplo, se hace un cálculo de lo que se gastó y lo que se ocupó en la fiesta anterior y saber en ese momento cuánto le toca a cada vecino, a veces 50 o 70 pesos cada quien, depende de los precios que en ese momento. Del precio de las cosas depende la cooperación que darán los vecinos, como el tiempo nos lleva, entonces se le aumenta.

La cuota es por familia, en el pueblo hay como sesenta nomás. Del dinero que se junta es para lo que alcance. (P. Díaz, entrevista, 2018)

Los vecinos cooperan, el presidente de Acción Católica que sea recibe el dinero y de ahí se va dando (a la cofradía); de acuerdo con una lista de cosas necesarias, como novillos, chile, verdura, manteca y otros, se hace un cálculo de los precios. En ocasiones, Acción Católica se encarga de comprar cohetones, manteca, chile y 
lo demás se le da al comité que va a sacar la fiesta. Días antes de la celebración, se hace la labranza de las ceras. En la iglesia, hay cajas de cera en bruto, las guarda Acción Católica, siempre se usa la misma cera. Los hombres son los encargados de dicha labranza.

[...] también se espera que llegue la Asociación Micaltepecana para ponernos de acuerdo, ellos vienen un mes antes de la fiesta, pero ya desde antes hicimos planes, casi cuando termina la fiesta ya tenemos pensado qué se puede hacer para el siguiente año, caminamos parejo con los paisanos. El día de la fiesta se hace un recibimiento a nuestros hermanos paisanos que no se olvidan de su pueblo ( L. Reyes.,entrevista, 2015).

El primer mayordomo es el encargado del recibimiento a los peregrinos, ya sean los paisanos radicados en el Distrito Federal o a las hermandades de los pueblos vecinos. El comité de la cofradía sale a recibirlos a la entrada de la comunidad, se hace acompañar de la banda de viento, así como de los integrantes de Acción Católica y del presidente auxiliar. El primer mayordomo les da la bienvenida en nombre de la comunidad, para luego entrar al pueblo en procesión hasta la iglesia. Se les recibe con un tono solemne y respetuoso. Se espera que los visitantes contesten del mismo modo.

Otra de las acciones de las autoridades religiosas es hacerse cargo de mantener la relación con otros pueblos, vía las hermandades. Estas son una forma de relación social voluntaria organizada en grupos locales y basada en la comunidad de creencias del catolicismo. De la misma manera coordina las correspondencias; es una de las principales instituciones de apoyo entre los pueblos mixtecos cuya principal finalidad es "hacer más grande la fiesta del pueblo":

Para la correspondencia lo que hacemos, es que si ellos vienen nosotros tenemos que ir de ley, porque ellos no van a preguntar si queremos, sino que ellos se organizan allí, por ejemplo; nosotros nos organizamos, porque ahora vino San Miguel, nosotros nos organizamos y vamos a tocar a San Miguel para que en la fiesta de nosotros vengan ellos. Nos organizamos y nos vamos, porque la costumbre de los pueblos es (que) llegando allá y lo primero que es visitar la iglesia, es lo primero (L. Reyes, entrevista, 2018)

Para la fiesta patronal, Acción Católica asesora y coordina las actividades de la cofradía. Es el primer vínculo con las autoridades civiles o religiosas (Asociación Micaltepecana, hermandades, etcétera). La cofradía es la directamente encargada de "sacar la fiesta", principalmente, la compra de suministros, preparación de los alimentos y atención a los peregrinos, además de la labranza de ceras. La Asociación 
Micaltepecana se ocupa de solicitar cooperación a los paisanos radicados en México y en Estados Unidos, así como de coordinar los eventos paganos. Las hermandades, en sus pueblos, organizan una colecta para apoyar la festividad; los vecinos del pueblo cooperan con una cuota de 1000 pesos. Las correspondencias apoyan con la música de viento u otra actividad como danzas o maromas.

Acción Católica, la presidencia auxiliar, la cofradía, las hermandades, las correspondencias, los vecinos del pueblo, los paisanos radicados en Estados Unidos y la Asociación Micaltepecana se unen en un esfuerzo común en apoyo a la festividad, para cubrir los diversos aspectos de esta.

Los mixtecos de El Rosario, a diferencia de los que viven en la Mixteca Alta Oaxaqueña, no desarrollan el sistema de mayordomías, donde algunos autores plantean que hay un proceso de redistribución de la riqueza, por el procedimiento nivelador de consumir el excedente monetario y en especie de los patrocinadores acomodados, en el cual los gastos de "cohetes, música, comida y bebida los paga el mayordomo en turno" (Butterworth, 1971, p. 129). La realización de la fiesta no sugiere un proceso nivelador.

\section{La Asociación Micaltepecana "nuevo actor”}

Esta asociación expresa un fuerte vínculo entre los radicados en El Rosario y quienes periódicamente regresan al pueblo. La mesa directiva tiene sede en el Distrito Federal. El Sr. T. C. nos explica la forma en que trabajan y cómo eligen a sus autoridades:

Con ellos trabajamos para dos cosas; uno para mejora del pueblo y otro para las festividades conmemorativas de aquí de la comunidad, que es en sí dos fiestas al año, la del Señor de la Salud en esta temporada de cuaresma y la de la Virgen de El Rosario que es la patrona del pueblo.

Se ha venido dando de manera voluntaria, tenemos que acudir nosotros a la ciudad de México a convocar a una asamblea y de ahí pues al igual que nos nombraron a nosotros por elección. ¡La mesa directiva está compuesta por el presidente, el secretario, el tesorero y tres vocales, aunque ahorita entraron cuatro... cinco! No, son cuatro vocales, pero el otro ya fue de manera voluntaria, el otro dijo, yo quiero formar parte también de la mesa, pero sí, son tres normalmente.

Para las ayudas con los paisanos radicados en Estados Unidos, la solicitud normalmente es vía la familia y vía mesa directiva, de acuerdo con quién lo solicite, normalmente la presidencia no nos permite, se puede decir, solicitar ese tipo de apoyos con ellos, lo solicitan los comités. Los diversos comités, y en este caso, lo presidentes de los comités que tienen familiares allá, como el gasto que se hace es grande, normalmente 
en una festividad, piden este tipo de apoyos, al igual que la mesa directiva, como ellos se encargan de sufragar gran parte de la feria, de igual manera, mandan invitaciones a los radicados en el extranjero y bueno, ya es voluntario lo que aporten. Estas cooperaciones son voluntarias (T. Cruz, entrevista, 2016)

En la celebración de las fiestas religiosas anuales, la asociación desempeña un importante papel en la organización sociorreligiosa: los migrantes regresan a sus pueblos, por lo menos una vez al año. Invierten miles de pesos para "alegrar el pueblo un rato".

En la celebración, el pueblo se encargará, principalmente, de los aspectos religiosos y los migrantes, de los eventos profanos, como la carpa con expendio de bebidas alcohólicas, el baile, el jaripeo, los juegos pirotécnicos, los concursos deportivos y otros. En días de fiesta, el regreso de los migrantes es un evento colectivo y organizado. La Asociación Micaltepecana es la encargada de organizarlos. Sobre esta asociación, una de sus fundadoras nos dice:

En 1968 entré a trabajar en casa en la ciudad de México, éramos pocos los que habíamos llegado. Empezamos a platicar para que se diera algo para los festejos de la fiesta del pueblo, éramos pocos, unos diez. Juntamos ochenta pesos cada uno, comprábamos flores y otras cosas. El día de la fiesta llegábamos por la calle principal, llegamos por primera vez en servicio particular, no había carretera, después tratamos un autobús especial para venir. $\mathrm{Al}$ año siguiente aumentó un poquito más y más, y año con año siguió creciendo.

Todavía no vivían en Prolongación Santiago, poco a poco llegaron a vivir [...] yo no pude continuar organizando, pero se quedaron los otros. Luego, que se casaron, murieron, cambiaron de domicilio, por eso se dividieron. Otras personas que decidieron seguir adelante buscaron a los que tuvieran voluntad para seguir adelante. Esto se sigue haciendo hasta ahora (P. Díaz, entrevista, 2018).

Se establece una diferencia entre las acciones anteriores y las actuales:

Ahora es más el dinero que ganan, ya alcanza para más: flores, manteles y otras cooperaciones. Se ponían de acuerdo con Acción Católica, con la Banda de Viento, y entonces llegábamos a las seis o siete de la mañana por el camino viejo, la entrada era por el camino viejo que viene de Petlalcingo, pasando por San Isidro, el Ídolo, y ya llegábamos, después entraron los camiones, ya se dirigían a dejar las flores a la iglesia y de ahí se retiraban a sus casas.

Los que iniciaron vivían y trabajaban en diferentes colonias, los domingos se reunían en casa del señor Ezequiel o donde yo trabajaba en Parque Hundido, platicaba cómo 
le iban hacer [...] en 1969 o 1970 ofrecimos un mantel bordado y tejido en cuadrillé que hasta la fecha está ahí, yo lo bordé y lo ofrecí. Ya luego se le puso el nombre de "Asociación Micaltepecana de Beneficio Mutuo", nos reunimos por la fiesta, ya luego también se hicieron otras cosas, otras cooperaciones para ayudar al pueblo, para poder arreglarlo [...] Ahora ya está más grande, ya se trabaja con los que están en Estados Unidos, ellos pueden mandar más dinero [...] ( B. Reyes, entrevista, 2014)

La Asociación Micaltepecana en sus orígenes fue formada por mujeres, actualmente participan, pero no tienen cargos de representación. La organización, de manera formal, no es parte del sistema de cargos, en la práctica, integra una sección fundamental de las lógicas organizativas de los rosareños; es el mecanismo en que el sistema de cargos está conectado con actividades translocales y transnacionales.

Se trabaja de manera coordinada, la elección del presidente se realiza en el Distrito Federal y se da a conocer en el pueblo quién ha sido electo; hay una comunicación previa, pláticas en las cuales se prevén los posibles candidatos. La presidencia de la asociación es detentada por varones, solo en 1999 se formó una mesa directiva constituida por mujeres no nacidas en el pueblo. Del desempeño no se cuenta con más datos, más que ellas cumplieron con el cargo el año que les tocó.

La Asociación es un comité que está externamente, por ejemplo, los que están radicados en México, allá ellos forman su comité, pero de acuerdo con el pueblo de acá, con el presidente, vamos, con el ayuntamiento, vienen o ellos nombran o se engrupa la gente y hay que hablar con los que van a quedar, formar pues ese comité y de ahí tienen que ver, van trabajando parejos (H. Reyes, entrevista, 2016).

\section{Cambio y continuidad en los roles}

En los últimos años, con el proceso migratorio, el ámbito de las decisiones interactúa en un nuevo y dinámico complejo de relaciones sociales, las cuales no se focalizan solo en un punto. Tal ámbito es multilocal; vecinos del pueblo asentados en el Distrito Federal, en la comunidad misma y en los Estados Unidos.

[...] ellos son los que cooperan, pero por medio de teléfono, se les invita. El comité les habla y la familia igual, ¿sabes qué? Esto vamos a hacer en el pueblo y en los comités van a pasar ahí con ustedes invitándolos a que vamos a hacer esto, una cooperación o algo grande como fue el proyecto del panteón, ¿no?

Mucho, mucho ha cambiado, por ejemplo, anteriormente, se iban los paisanos, pero por medio de cartas. No nos comunicábamos, era muy difícil a que ellos se comunicaran por lo que hay de la familia de nosotros y a lo que había en el pueblo, 
a como ahora, porque ahora por teléfono, todo lo que hay acá, al rato se dan cuenta, ellos y anteriormente era poco, se puede decir, ¿no? Que por ejemplo ahí están mis hermanos, R. G., que eran tres nada más, pero hoy no, sino hoy se llaman entre ellos y se pasan entre ellos y se comunican, estamos más comunicativos, se puede decir, pero por medio del teléfono, pero, ¿por qué? Porque está un comité, de ellos, por ejemplo, se comunican tanto como nosotros, en este caso yo a mis hijos. Pero si yo le digo a mis hijos, ¿sabes qué? Van a dar tanto, ellos los van a invitar, pero mira hijo, yo no tengo, quiero que tú me ayudes. El también me tiene que dar y tiene que dar a lo de la mesa. Nosotros les decimos a los de la mesa, a los del comité, así no (H. Reyes, entrevista, 2016).

Esto ha traído una multiplicidad de maneras de vincularse; se han reconfigurado formas de designación para los cargos y cambios en los perfiles requeridos para los sustentantes, el hecho cualitativo es el nombramiento de una mujer como presidenta auxiliar, el posicionamiento de género en otras funciones y la consideración de las madres solteras como "vecinas activas" o ciudadanas. Todo ello nos habla de esos nuevos perfiles necesarios para estos métodos novedosos de vinculación política.

Las representaciones de género han cambiado, ha habido una resignificación para quien debe ocupar el cargo y esta cuestión no se responde solo vía el "desplome demográfico”. Existen varones con las características necesarias para ocupar los oficios, el mismo cabildo está conformado por varones; aun así, se realizó la designación de una mujer como autoridad principal. Lo que he querido apuntar son estas dinámicas novedosas que la experiencia migratoria está generando para el sostenimiento de la comunidad.

Nuevos actores entran en la trama social; el incremento del nivel educativo y el retorno de los migrantes crean condiciones para un cambio en los perfiles requeridos por la comunidad. Aquellas personas con mayores recursos económicos, cantidad de tierras y escolaridad se convierten en las aceptadas socialmente para ejercer los cargos civiles.

Se mantiene una actitud diferenciada en torno a los espacios de lo civil y religioso. Mientras que para un migrante era significativo aportar ayuda en pro de la fiesta y ser considerado responsable de la cofradía, para un integrante del magisterio tenía mayor relevancia ocupar un cargo político como presidente auxiliar.

Las funciones religiosas solian ser ocupadas, principalmente, por aquellas personas con poca tierra, tejedores con menor trayectoria migratoria. Esta redistribución entre quienes ocupaban los cargos civiles y religiosos va configurando el nuevo escenario de las representaciones colectivas en torno al quehacer político y la exigencia de un 
perfil diferenciado; mientras que lo concerniente a la construcción de las expresiones religiosas era el ámbito acotado para la gente situada en la base de la pirámide social. Actualmente, la presidencia de Acción Católica está al mando de un exmigrante.

El sistema de cargos ha pasado por una suerte de "prueba y error". Previo a que hubiese suficientes vecinos que detentaran los cargos (antes de la migración), las funciones civiles eran ejercidas, primordialmente, por quienes tenían mayor cantidad de tierras; los que contaban con menor número de terrenos y se dedicaban más que todo al tejido de sombrero de palma eran a quienes se les designaba para las ocupaciones religiosas.

En la década de los ochenta, fue cuando el perfil para los cargos civiles cambió, con preferencia hacia aquellas personas que tuvieran una formación en el magisterio; los cargos religiosos siguieron en manos de los tejedores y vecinos con poca tierra. Ante la salida de una gran cantidad de familias (del D. F., desde la década de los 60, y de EE. UU., en los 90), la elección de autoridades civiles se dinamiza, el sistema de cargos entra en crisis y las mujeres antes exentas de puestos deben asumir algunos de ellos. En la primera década del 2000 , se presencia un cambio, se rompe con toda una tradición de presidentes auxiliares varones y las féminas irrumpen en la esfera pública, recaen en algunas parte de los nombramientos. El marco de acción de la presidenta encuentra un terreno favorable, al contar con el apoyo de sus redes de parentesco; sus hermanos, hijos, y amigos establecidos en Nueva York le respaldan económicamente. El sistema de cargos está conectado y opera mediante actividades translocales y transnacionales.

Ser mujer y ser autoridad trasciende en la vida de la comunidad. Herlinda, como actora social, tiene la claridad de que está en un momento sustancial de la comunidad, avalada por el presidente municipal, el cual está satisfecho por su condición de mujer, figura que va a capitalizar para obtener recursos de programas gubernamentales como Sedesol, CDI y ramos 28 y 33. Ella acepta con resignación cuando al término de su gestión es nombrada servicial. Paralelo a ello, surge la descalificación, por parte de los varones, a su gestión como presidenta y la de su tesorera; las frases que expresaban la perspectiva masculina a la gestión de ambas mujeres eran: "solo salen a buscar hombres" o "pinche vieja, ¿cree que es muy fácil ser autoridad?" (H. Reyes, entrevista, 2016).

Parte de los cambios los observamos en las maneras con las cuales se incorporaron al sistema los paisanos; esto podría traducirse a que, con la migración, la modalidad de "envío de ayudas económicas" supliera la ausencia física de aquellas personas que de estar en el pueblo tendrían que asumir ese aporte. La situación se complejiza, a pesar de que la materialización de los apoyos es clara; entre ellos están ayudas económicas 
para la fiesta; alumbrado público; obras como el arreglo de la presidencia, la barda del panteón o la iglesia del pueblo; decisiones para apoyar tal o cual cosa, que pasan por un cabildeo dado de cuando menos dos maneras: 1) la comunicación verbal con los familiares e interesados en una labor o 2 ) vía oficio (entre los paisanos es discutido y se toman decisiones).

La categoría de paisano representa a un ciudadano, un integrante del pueblo que se halla fuera del lugar, pero que forma parte de la comunidad. La membresía la garantiza o la ejerce por medio de los servicios a su pueblo. La Sra. H. R nos dice qué es lo que se considera un ciudadano:

[...] para ser un ciudadano del pueblo, tú tienes que cooperar con el pueblo, en primera, dar tu cooperación, dar tu faena, estar pendiente por ejemplo que se nos ofrezca tequio, faena, lo que sea, tienes que estar pues en eso, ahí es ser un ciudadano, cuando tú quieras, proponer algo, que tú digas: yo propongo, o mi opinión es esta, ¿qué dicen señores vecinos? ¿No? Ahí no te puedo decir nada, ¿por qué? Porque tú estás cooperando, estás pues con el pueblo, pero si mientras tú no tienes cargo no quieres cooperar con el pueblo, eso es no ser un ciudadano, pues (H. Reyes, entrevista, 2010).

\section{Conclusiones}

El sistema de gobernanza de las comunidades indígenas difiere de las nociones que descansan sobre conceptos vitales como la ciudadanía en el derecho positivo, porque, a diferencia de su condición pasiva en este, ella radica, en aquel, en un constante ejercicio de transformación con dinámicas como la migración interna e internacional. La ciudadanía sustantiva ha reposado sobre una concepción de comunidad homogénea, con sistemas de pertenencia únicos, sin reparar o incluir en el análisis la diversidad de actores y arenas de contiendas que existen en su interior. El tránsito hacia la ciudadanía multicultural es una de las próximas tareas en la construcción de políticas públicas del Estado mexicano.

Asimismo, en la visión tradicional está presente el tema de la ciudadanía, al basar su concepción en términos de un estatus personal, dotado de derechos y deberes individuales; pero esta noción se topa con una realidad contrastante, cuando hablamos de pueblos indígenas. Aun así, se procura el equilibrio entre los derechos individuales y colectivos. Por su parte, el rumbo de la gobernanza no es algo abstracto, de su adecuación depende la supervivencia de esos núcleos poblacionales, excluidos de las políticas públicas de distribución del gasto gubernamental, vía infraestructura y dotación de programas de bienestar, que se ven obligados a construir estrategias de desarrollo autónomas. En este esfuerzo, concurren los diversos actores, como es el caso de los migrantes, razón por la cual el mecanismo de nombramiento debe 
readecuarse para integrarlos como sujetos de derecho. Por otra parte, pese a las innovaciones y los cambios ocurridos en el sistema de nombramiento, aún quedan temas torales pendientes como el género y la diversidad religiosa; además, todavía se mantienen prácticas de exclusión para las mujeres y minorías religiosas.

\section{Referencias}

Butterworth, D. (1971). Migración rural-urbana en América Latina: el estado de nuestro conocimiento. América Indígena, XXXI(1), 85-105.

Kearney, M. y Besserer, F. (2004). “Oaxacan Municipal Governance in Transnational Context.”, en Indigenous Mexican Migrants in the United States. J. Fox and G. Rivera-Salgado, editores. Centro de Estudios México - Estados Unidos, Universidad de California, San Diego, pp. 449-466.

Hernández, E. (1996). Vocabulario en lengua castellana y mexicana de fray Alonso de Molina. España: Consejo Superior de Investigaciones Científicas.

INI. (1998). Los sistemas normativos de las comunidades indígenas en Chiapas. [Documento]. Chiapas, San Cristóbal de las Casas: INI. 